\title{
Proposed system for measuring the vertical cold-mass motions in the RHIC cryogenic quadrupoles
}

\author{
P. Thieberger, W.W. MacKay, V.I. Ptitsyn, D. Trbojevic
}

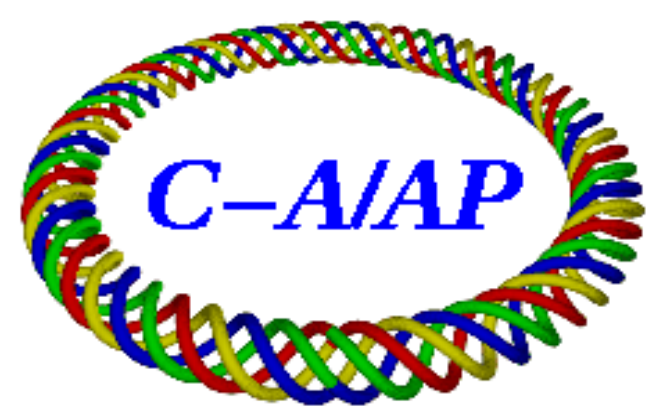

Collider-Accelerator Department Brookhaven National Laboratory Upton, NY 11973 


\title{
Proposed system for measuring the vertical cold-mass motions in the RHIC cryogenic quadrupoles
}

\author{
P. Thieberger, W.W. Mackay, V.I. Ptitsyn and D. Trbojevic
}

\section{Introduction}

During the 2005 RHIC run, the important observation was made that the beam closed orbit experiences diurnal variations, correlated with the outside temperature variation cycle [1]. This is suspected to be one of the main factors affecting the machine (and beam lifetime) reproducibility. According to the analysis of the closed orbit variations, the sources of the variations are located in the interaction region triplets, with the largest source being at the IR4 triplets. Since the observed orbit variation is largest in the vertical plane, direct measurements of the vertical motion of IR quadrupoles should provide deeper insight in the origin of the problem.

Since the IR quadrupoles are cryogenic, the magnets being hidden inside the cryostat, the direct measurement of the cold mass positions is not an easy task. Because of this, more straightforward measurements, which observe the vertical displacement of at different exterior points of the RHIC IR magnet cryostats were made first [2]. These measurements indicate that there may be sufficient motion of the cold masses to produce the beam orbit variation observed during the run. However measurements performed at different heights differ drastically making it difficult to estimate the corresponding displacements of the cryogenic lenses. The magnitude of the measured exterior displacements varies between tens of microns and $\sim 2 \mathrm{~mm}$. These motions are approximately periodic with a period of 24 hours correlating well with the diurnal thermal cycle. This correlation has recently been confirmed with sensitive inclinometer measurements [3].

\section{Proposed method}

In view of the above facts it becomes desirable to perform direct measurements of the cold-mass positions as a function of time. To this end we propose to develop a system based on the absorption of a well collimated beam of gamma rays partially intercepted by the outer iron surface of the cold mass. The transmitted intensity as measured by a detector will then be modulated by the motion of this absorber. Since this motion is very slow, it should be possible to accumulate sufficient counting statistics to detect displacements that are small compared with the size of the gamma-ray beam, and to do this without the need of very high counting rates or very strong sources.

In Fig. 1 we show a schematic diagram illustrating the principle of these measurements. Gamma rays from a source located at the left of the cryostat are detected through a collimator in a detector located at the right side in such a way that the gamma ray beam defined by the source and collimator is partially intercepted by one of the two cold masses to be monitored. To miss the other cold mass and its blanket a $\sim 7^{\circ}$ slope is indicated for the gamma ray beam.

During setup the gamma-ray source and detector can be simultaneously displaced by equal increments, until the desired operating point is reached as shown on the chart to the right. The slope of counting-rate vs. position is determined, and can be used for one of the two operating modes to be 
described next. The small contribution to this slope from the traversed thickness variations with height of the materials other than the cold-mass-blanket interface turns out to be negligible but could if necessary be applied as a small correction.

The first measuring method, depicted by the chart at the bottom left of Fig. 1, consists simply in leaving source and detector stationary and following the counting rate as a function of time. The advantage of this method is its simplicity and cheaper positioning stages, but it depends on the calibration described above, and that calibration is only approximately linear over a limited range.

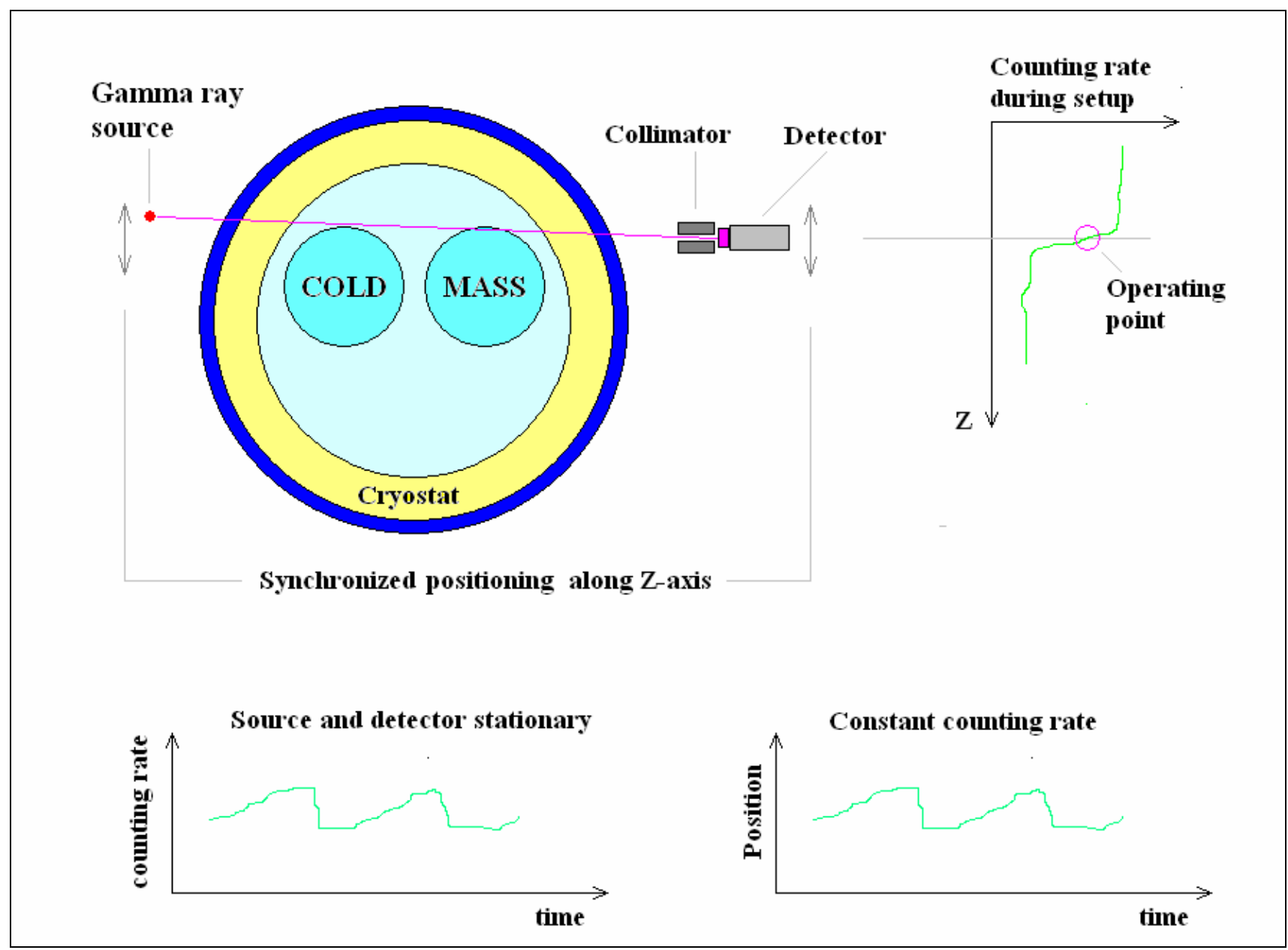

Fig. 1 Schematic diagram of the arrangement with illustrations of two types of measurements shown at the bottom (see text).

In the second method, depicted by the chart at the bottom right, the average counting rate is kept constant by closing a feedback loop that simultaneously displaces source and detector to compensate for the motion of the cold-mass. Source and detector will then be forced to follow the displacements that are being measured, and their own displacements are recorded. This is somewhat more involved but no calibration is required other than knowing the characteristics of stepper motors or linear 
encoders, and the system is linear over its entire range. The required resolution of the vertical motion control needs to be better and will be more expensive.

We will use a $\mathrm{Co}^{60}$ source because of the good penetration of its relatively high energy gamma rays (1.17 and $1.33 \mathrm{MeV}$ ) and because of the relatively long half-live (2.7 years). Figure 2 shows a typical pulse-height spectrum from this source recorded in a 3" long, 3" diameter NaI(Tl) scintillation crystal mounted on a photomultiplier tube. The discriminator setting will be chosen as indicated selecting the photo peaks to discriminate against scattered radiation and so as to minimize effects of any drifts in the peak positions. The position of the peaks and discrimination level will be adjusted and monitored with the help of a multichannel pulse height analyzer (MCA) as explained later.

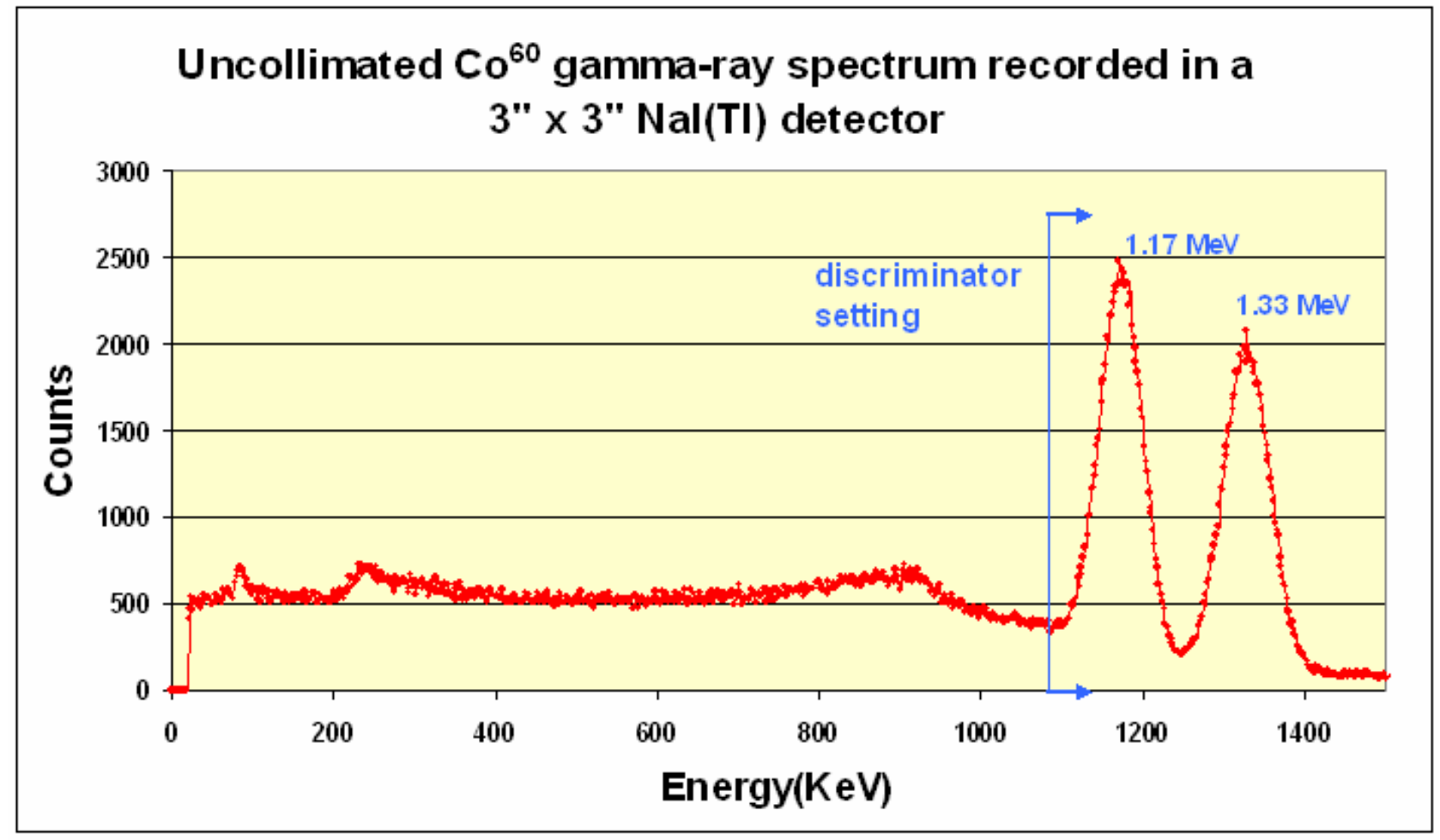

Fig.2

\section{Mechanical design}

The left side of Fig.3 shows a roughly to-scale view of a possible physical arrangement of source collimator and detector. In this example the $20 \mathrm{mCi} \mathrm{Co}^{60}$ source is a needle or "seed", $0.25 \mathrm{~mm}$ in diameter horizontally aligned (perpendicular to the plane of the drawing) so as to minimize its contribution to the width of the collimated gamma-ray beam. That beam is defined by the source and the $3 \mathrm{~mm}$ wide lead collimator slot in front of the detector. The vertical width of this beam at the cold mass measuring point is about $1 \mathrm{~mm}$. The lead collimator surrounding the source is a few mm wide and its only purpose is radiation protection and the reduction of scattered radiation. The shutter is also for radiation protection while the equipment is not being used and while the source is being transported. 
The reference plane for these motion measurements can be the concrete floor which is thought to be adequately stable [4]. Since we are interested in a $\sim 10 \mu$ precision, we must consider the thermal expansion of the vertical supports. For a $2 \mathrm{~m}$ height and a $5^{0} \mathrm{C}$ temperature variation, the upper platform will move by $\sim 170 \mu$ if the supports are made out of steel and $\sim 250 \mu$ if they are aluminum. Even for a low expansion material such as Super Invar that is probably too expensive to use, the displacement would still be $\sim 6 \mu$ for a $5^{0} \mathrm{C}$ temperature swing.

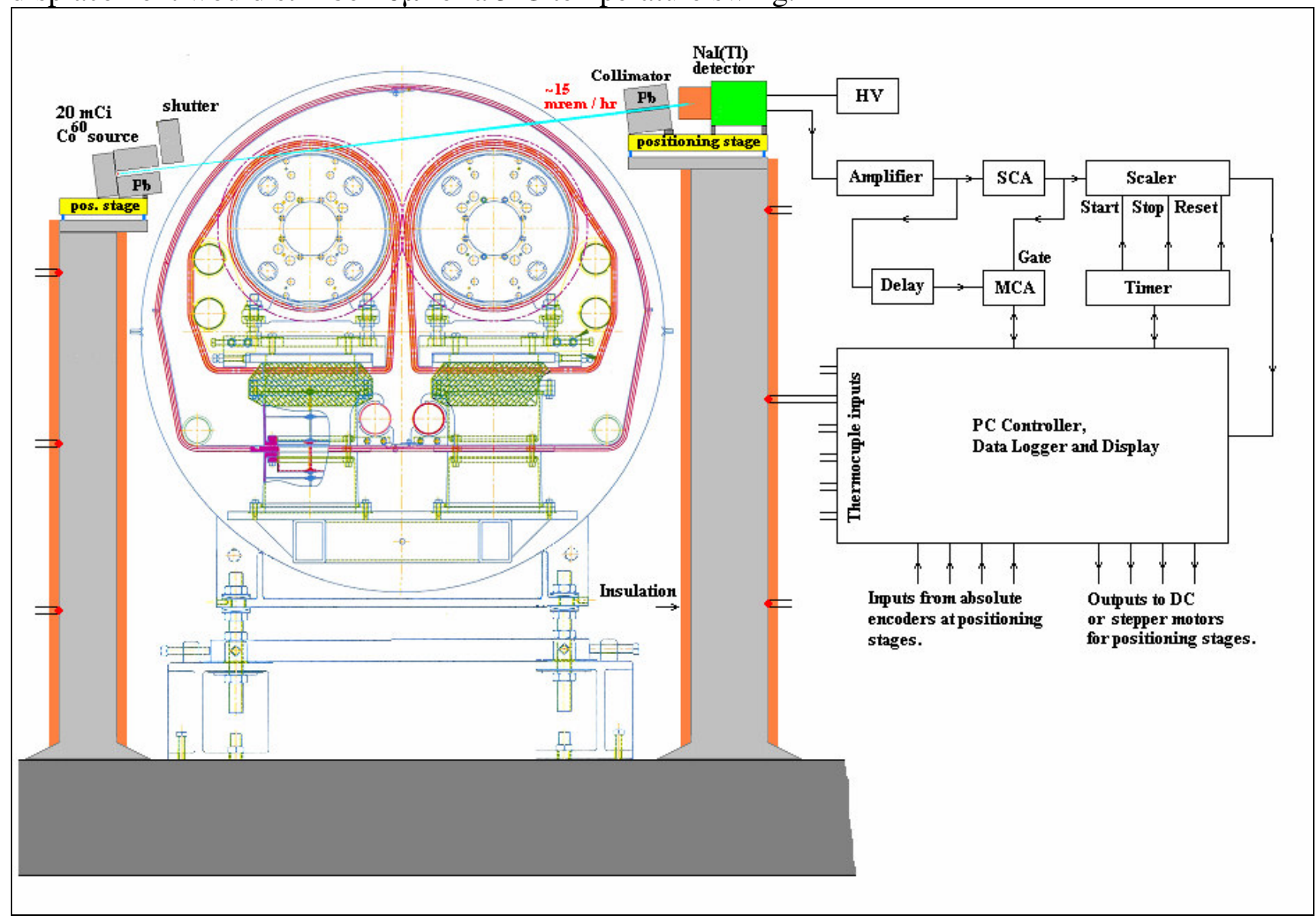

Fig 3 Conceptual design of the hardware and associated instrumentation and controls (see text).

One solution is to carefully monitor the temperature of the vertical supports at several locations and apply the corresponding corrections to the data. A precision of $\pm 0.1{ }^{0} \mathrm{C}$ would lead to a $\pm 3 \mu$ error, which should be acceptable. The thermal insulation shown on the supports together with the good thermal conductivity of e.g. aluminum will assure sufficient temperature uniformity along each support.

The other solution to deal with the thermal expansion of the supports is to monitor and record the positions of the upper platforms by using wall-mounted optical systems like the ones used to detect cryostat motions [2]. It may make sense to use both systems simultaneously since this thermal expansion problem will probably be the main factor limiting the accuracy of these measurements. 


\section{Instrumentation, data acquisition and controls}

The right side of Fig. 3 shows a block diagram for the data acquisition and control system. The detector consists of a 3 " diameter $\times 3$ " length cylindrical $\mathrm{NaI}(\mathrm{Tl})$ crystal optically coupled to a photomultiplier tube. The High voltage supply (HV), the amplifier and the Single Channel Analyzer (SCA) should be stable enough to guarantee a discrimination level constant to $\pm 0.1 \%$ of the photopeak height (see Fig. 2). The position of the discrimination level relative to the photo peaks is adjusted and monitored by means of a Multichannel Analyzer (MCA) gated by the SCA output. MCA spectra can also be periodically stored for verification and data correction if necessary. By automatically monitoring peak positions, it is also possible to proveide feedback to the detector high voltage so as to keep the positions constant, but this should not be necessary.

Data collection consists in recording sequential, time-stamped, scaler counts after constant counting periods determined by the timer. Variations in these counts are then translated into position data according to a calibration performed at the beginning of the run

Alternatively, the scaler counts can be compared to a fixed number, generating feedback to reposition source and detector so as to keep the counting rate constant. In this second case the stored data consists of time-stamped positions. In both cases thermocouple data are also stored and used to apply corrections to the position data.

The controller, data logger and display can all be implemented in a Personal Computer (PC). Depending on the available electronics, some of the other devices shown in the block diagram of Fig. 3 , for example the scaler, timer and MCA may be implemented as PC plug-in cards. The software development for this system will be a major part of the effort.

\section{Expected performance}

An important aspect for evaluating the performance of this system is the time it will take to accumulate sufficient number of counts for reaching the required statistical accuracy of each point. The relevant factors are the source strength, the solid angle defined by source and collimator, the photo-peak efficiency of the detector, and the gamma-ray absorption in the materials other than the object of interest that the radiation must traverse. Starting with this last question we use the information shown in Fig. 5 and in Table 1 to estimate this absorption. 


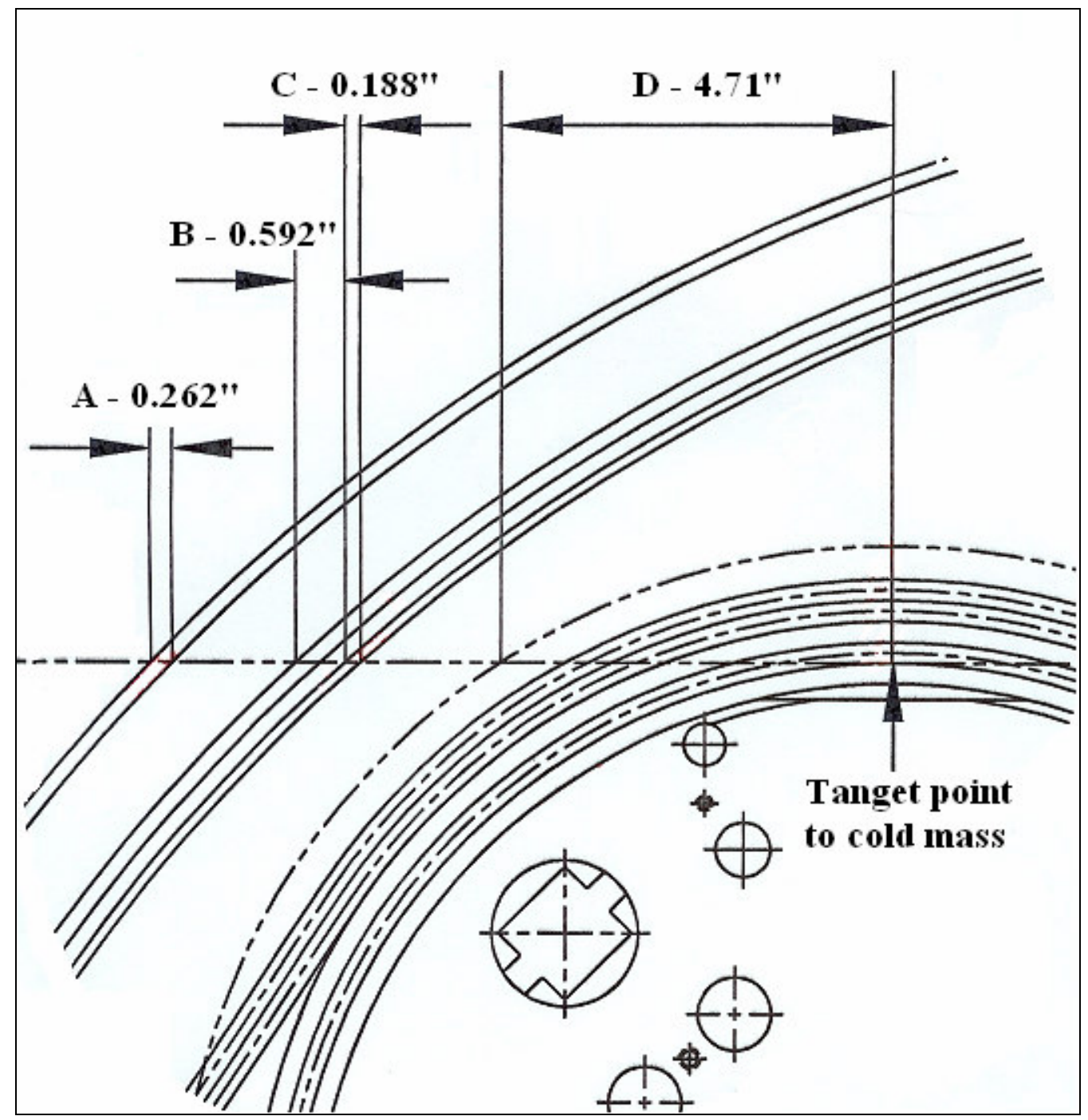

Fig. 5 Sketch indicating materials that are intercepted by a $\gamma$-ray tangent to the cold mass (see table 1). For simplicity traversed thicknesses were calculated for a horizontal trajectory, while the measurements will be performed at a small $\left(\sim 7^{0}\right)$ inclination (see Figs. 1 and 2$)$. The small errors thus introduced by the fact that the circles are not concentric are totally negligible for the present estimates.

Table 1. Materials traversed from outside of cryostat to the cold-mass tangent point

\begin{tabular}{|l|l|c|c|c|}
\hline Item & $\begin{array}{c}\text { Distance } \\
\text { traversed }\end{array}$ & $\begin{array}{c}\text { Average } \\
\text { density }\end{array}$ & $\begin{array}{c}\text { Average mass attenuation } \\
\text { coefficient for } \mathrm{Co}^{60} \gamma \mathrm{s}\end{array}$ \\
\hline & & $(\mathrm{cm})$ & $\left(\mathrm{g} / \mathrm{cm}^{3}\right)$ & $\left(\mathrm{cm}^{2} / \mathrm{g}\right)$ \\
\hline A & Cryostat (steel) & 0.67 & 7.87 & $5.43 \mathrm{E}-2$ \\
\hline B & Heat shield blankets & 1.50 & 0.415 & $5.65 \mathrm{E}-2$ \\
\hline C & Heat shield (aluminum) & 0.48 & 2.70 & $5.55 \mathrm{E}-2$ \\
\hline D & Magnet blankets & 12.0 & 0.553 & $5.65 \mathrm{E}-2$ \\
\hline
\end{tabular}


Using the data of Table 1 we calculate a transmission of $21.6 \%$ for a gamma-ray trajectory tangent to the cold mass. This number will not be significantly affected should the outer shells, i.e. the cryostat, heat shield and heat shield blankets move with respect to the cold-mass and its blankets even by several $\mathrm{mm}$. The small variation which is only due to the curvature of these outer shells is of second and higher orders in the displacement and totally negligible for our purposes.

Next, to see what happens when the cold mass gradually intercepts increasing portions of the beam, we performed numerical simulations of transmission as function of position for $\mathrm{Co}^{60}$ gamma-ray beams collimated to $0.1 \mathrm{~mm}, 1 \mathrm{~mm}, 2 \mathrm{~mm}$ and $3 \mathrm{~mm}$. For this purpose the wider beams were subdivided into $0.1 \mathrm{~mm}$ slices and mass attenuation coefficients were used for the magnet steel and for the blanket material averaged over the two gamma energies $1.17 \mathrm{MeV}$ and $1.33 \mathrm{MeV}$. This averaging is justified because of the close proximity of the values. Figure 6 shows the result of this simulation which was performed with an Excel spread sheet. Transmission is plotted as function of position. The zero of the position scale corresponds to the center of the gamma-beam being tangent to the cold mass surface.

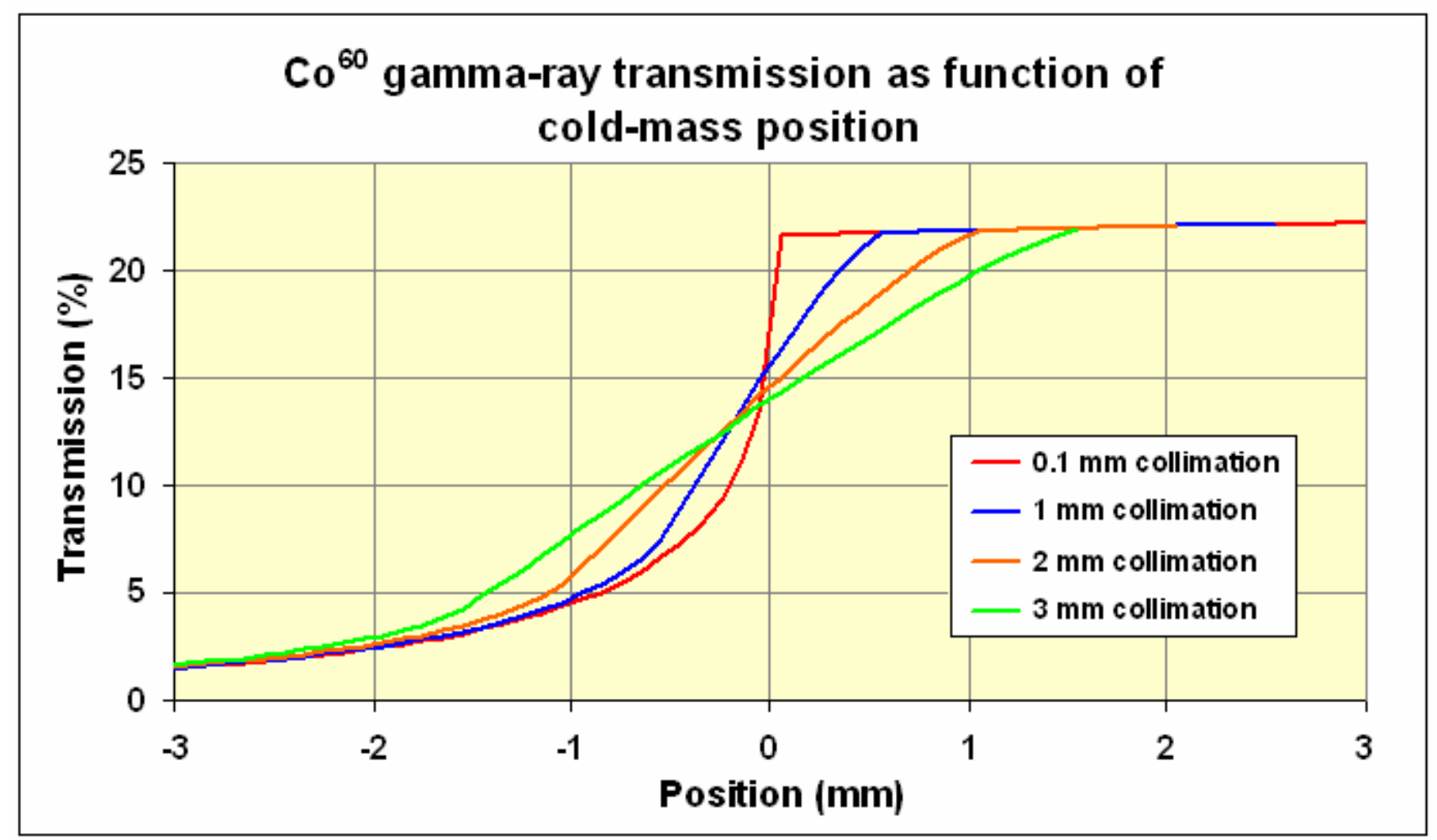

Fig. 6 
We see from Fig. 6 that, as expected, a very narrow collimation can provide a large slope and therefore great position sensitivity. The approximately linear region is however very narrow thus providing a very small dynamic range that is unacceptable, at least for the open loop system described before. To obtain a reasonable dynamic range we will select $1 \mathrm{~mm}$ collimation or larger, and an operating point roughly in the middle of the approximately linear parts of the curves, i.e. around the $13 \%$ transmission points which correspond roughly to a $40 \%$ reduction of the maximum counting rate..

For these operating points, the sensitivity in terms of $\%$ counting rate change per 10 micron motion is plotted in Fig.7 as function of collimation (black curve and left-side scale). The numbers of counts required to obtain statistical rms accuracies of 10 microns are plotted as the red curve with the corresponding right side scale

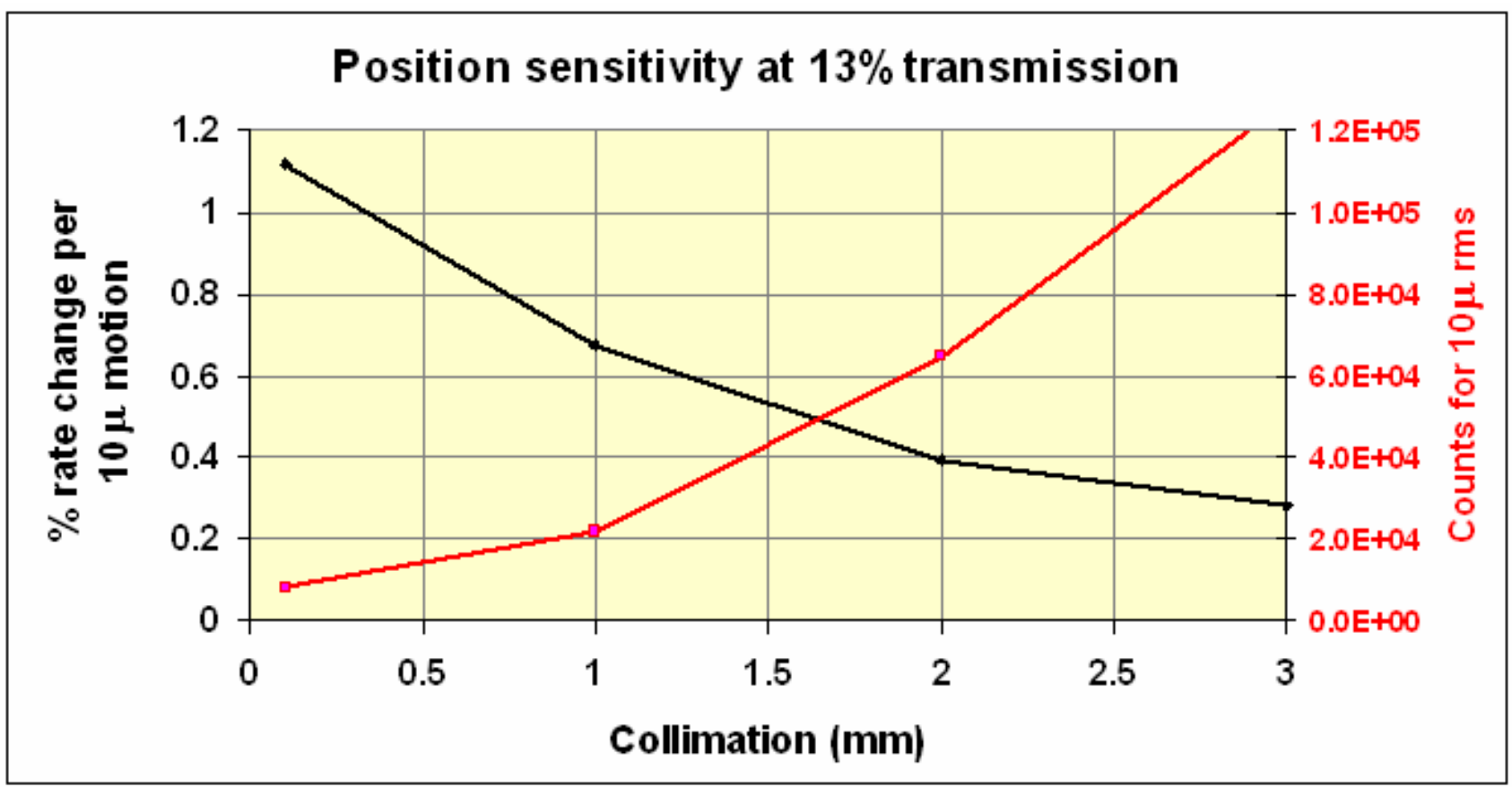

Fig. 7

Now, adopting the $1 \mathrm{~mm}$ collimation case as an example, we can determine the counting times and the radiation levels for 10 micron rms measurements as a function of source strength.. The results are shown in Fig. 8. We see that for a $20 \mathrm{mCi}$ source the counting time will be $\sim 40 \mathrm{~s}$, and the radiation level at the detector side will be $\sim 15 \mathrm{mrem} / \mathrm{hr}$. Given the very slow displacements, a 1 minute measuring time seems more than adequate, and $15 \mathrm{mrem} / \mathrm{hr}$ is compatible with a low radiation area $(<100 \mathrm{mrem} / \mathrm{hr})$. In principle it should be possible to perform these measurements even during RHIC operation if sufficient shielding can be provided for the detector. 


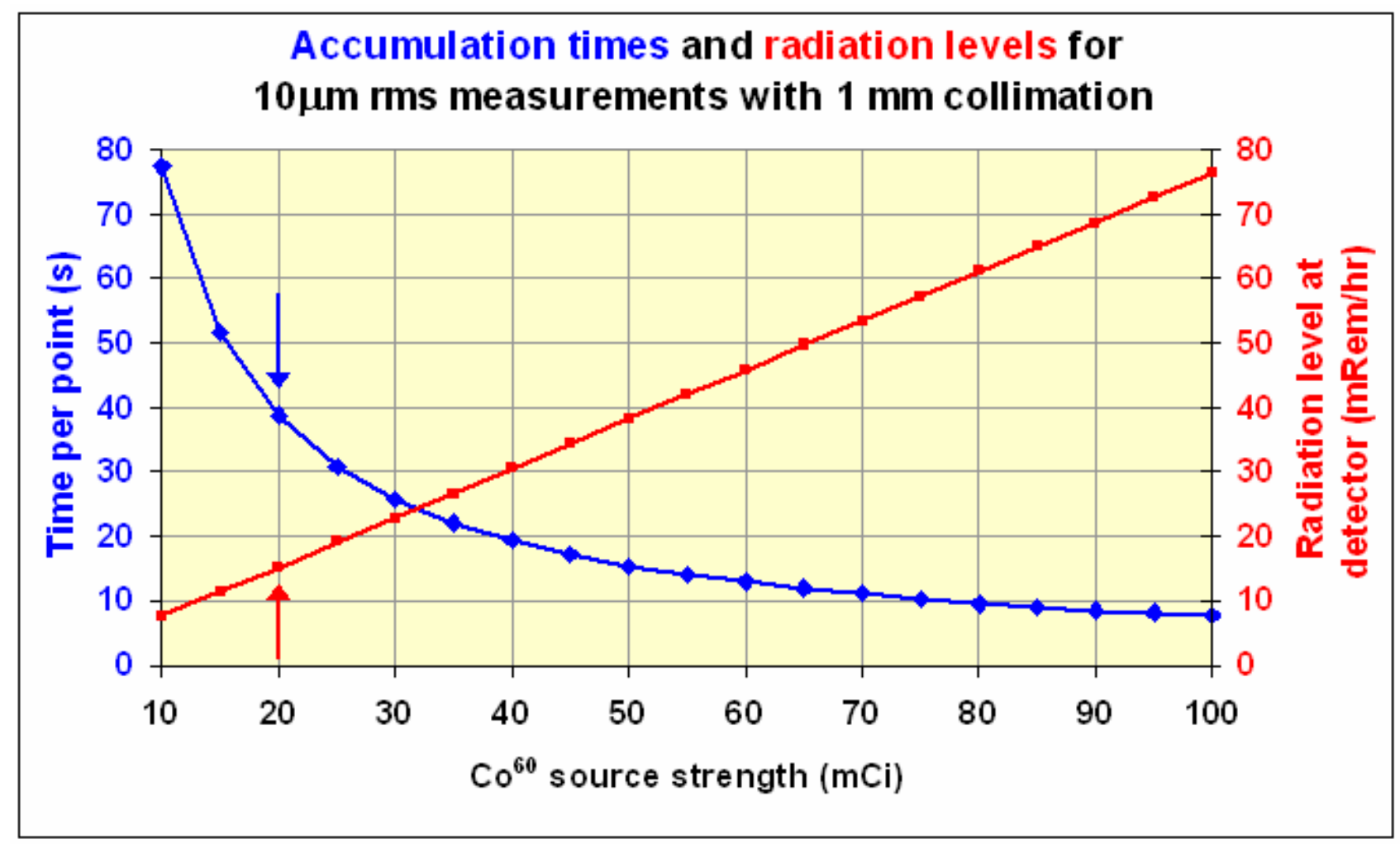

Fig. 8

\section{$\mathrm{Co}^{60}$ source and radiation safety issues}

As discussed above, the dose rates in the vicinity of the detector will be around $15 \mathrm{mrem} / \mathrm{hr}$. The area where measurable radiation is present is limited by collimating the radiation form the source (see Fig. 2). There will be a small high radiation volume at the exit of the source collimator, but limited to a relatively inaccessible area between source and cryostat. The source collimator will be provided with a shutter that will be closed when no measurement is being performed and during transportation of the source. Remote operation of this shutter is not necessary, but it can be provided if desired.

The $20 \mathrm{mCi} \mathrm{Co}{ }^{60}$ source will be in the form of a thin cobalt wire perhaps $0.25 \mathrm{~mm}$ thick and $5 \mathrm{~mm}$ long that has been activated in a reactor. It is important for this source to be thin in order to provide a narrowly collimated beam defined by the source and a remote collimator. The company providing these sources [5] nickel plates the needles after irradiation so that they become non-contaminating. A secondary barrier will be provided, either by inserting the wire in a piece o thin hypodermic tubing with sealed ends, or by placing it in a narrow machined slot covered with an aluminum plate. This plate can be sealed (o-ring or epoxy) but this isn't really necessary since the secondary containment only needs to provide physical protection to the non-contaminating source. Similar sources [6] are being used at CERN for calibration of the CMS Hadron Calorimeter for the LHC. 


\section{Summary and Conclusions}

The problem of monitoring small displacements of the cold-masses in RHIC quadrupoles can be solved by shining gamma-rays through the cryostat. Modeling of the absorption as function of position of a well collimated gamma-ray beam shows that one measurement per minute with a 10 $\mu \mathrm{rms}$ statistical error can be obtained with a $20 \mathrm{mCi} \mathrm{Co}^{60}$ source. A rather detailed conceptual design is presented. The main potential source of systematic errors will be the thermal expansion of the supports, but careful monitoring of their temperatures will probably suffice to apply the required corrections. Radiation safety issues were considered and found to be relatively benign.

\section{Acknowledgement}

One of us (P.T.) would like to thank Dr. Mario Mariscotti at Tomografía de Hormigón Armado, S.A., Buenos Aires, Argentina, for introducing him many years ago to this general type of measurements, for continuing collaboration, and for recent generous comments.

\section{References}

1) Observations done by V.Ptitsyn, D.Bruno and J.Morris. See RHIC Retreat 2005, "Orbit mysteries:clues and cures".

2) D. Trbojevic, et. al. to be published.

3) D. Bruno, V.I. Ptitsyn, C. Montag, in progress, to be published.

4) Stability of floor as reference surface. D. Trbojevic?

5) North American Scientific, 20200 Sunburst Street, Chatsworth, California 91311

Phones: (800) 992-6274, 818-734-8600 (FAX 8606).

6) http://sx5-hcal.web.cern.ch/sx5-hcal/rad/files/Rad_saf.doc. 